\title{
Detection of Biomarkers Using LSPR Substrate with Gold Nanoparticle Array
}

\author{
Young Min Bae, Seung Oh Jin, Insoo Kim, Ki Young Shin, \\ Duchang Heo, and Dong-Goo Kang
}

Korea Electrotechnology Research Institute, 111 Hanggaul-ro, Ansan 426-170, Republic of Korea

Correspondence should be addressed to Young Min Bae; kimbym@keri.re.kr

Received 3 July 2015; Revised 8 September 2015; Accepted 13 September 2015

Academic Editor: Fangfang Sun

Copyright (c) 2015 Young Min Bae et al. This is an open access article distributed under the Creative Commons Attribution License, which permits unrestricted use, distribution, and reproduction in any medium, provided the original work is properly cited.

\begin{abstract}
In the biosensing platform, label-free detection technique provides advantages such as the short analysis time and the costeffectiveness. In this study, we showed the feasibility of the LSPR substrate with gold nanoparticle array for detecting low density lipoprotein (LDL) and high density lipoprotein (HDL) without labeling. The LSPR substrate was fabricated through the lift-off process with the anodized alumina mask, and its LSPR phenomenon was observed by measuring the optical transmission of substrate. The antibodies were immobilized on the gold nanoparticle array via the chemical binding, in which the 11-MUA was used as the linker to bind the antibodies. The binding of antibodies was confirmed by observing the shift of LSPR peak of the substrate. Finally, with the LSPR substrates with the antibodies immobilized, the detection of LDL and HDL was investigated. As a result, LDL and HDL could be detected in the clinically available concentration range, respectively.
\end{abstract}

\section{Introduction}

Immunoassay based on antigen-antibody binding is prevalently used for detecting several kinds of analytes such as protein, pathogens, or small molecules. As the method can be quite rapid, it has been applied to develop a point-of-care diagnostic device of which the operation should be simply implemented [1].

The labeling-based detection techniques have been widely employed for detecting the antibody-antigen binding in the point-of-care diagnostic device $[1,2]$. For example, a fluorescence detection technique has been used to detect antibody-antigen binding on a solid surface. In the technique, the labeling step, in which the complex of antibody-antigen binding is labeled with the probe antibody, conjugated with fluorescence molecule. Basically, the labeling techniques involving the use of fluorescence, ELISA, and isotropic labeling require one or two intermediate steps for labeling. So, the simpler detection technique should be developed for implementing the point-of-care diagnostic device.
Label-free detection technique would provide a direct approach to the detection of protein-protein binding. Electrochemical impedance technique, surface plasmon resonance (SPR), and mass-sensitive cantilever detection have all been applied to label-free detection methods [3-5]. In particular, the SPR technique has been used as a versatile detection tool for the study of the kinetics of receptorligand interaction [6]. In the conventional SPR technique, the plasmon resonance is excited by the light beam incident onto the interface between a metal and a dielectric medium, and the plasmon resonance condition such as the incident angle is attributed to the refractive index in the dielectric medium. So, the complicated optical system or mechanical structure is required to implement the SPR device [7]. On the other hands, the localized surface plasmon resonance (LSPR) is observed in the metal nanoparticle, not presented in the bulk metal [8]. When light is incident on a surface with metal nanoparticles smaller than the wavelength of light, the collective oscillation of conduction electron in them with a resonant frequency is induced. The LSPR is 
dependent on the dielectric properties of the local environment surrounding the nanoparticles [9]. This property allows the LSPR to provide the label-free detection method for an antigen-antibody reaction [10]. Also, as the LSPR of metal nanoparticle is easily observed by measuring the adsorption spectrum, the biosensor platforms based on the LSPR can be simply implemented.

The substrate, onto which the nanosized metal structures exhibiting the LSPR phenomenon are deposited, should be developed for the LSPR-based biosensor. There have been several methods reported to fabricate the LSPR substrate, such as the direct deposition of nanoparticles or nanorods in solution onto a solid surface, the growth of nanoparticles on the surface, nanosphere lithography, and nanoimprint lithography [9-14]. Recently, we reported the fabrication of gold nanoparticle array on solid surface [15]. The gold nanoparticle arrays were simply fabricated via the lift-off process, and the absorption peak showing the maximum absorbance in the absorption spectrum (defined as the LSPR peak) could be regulated in the wavelength range of $500 \sim 700 \mathrm{~nm}$ with the control of thickness.

In this study, we demonstrate the application of the gold nanoparticle array fabricated by the lift-off process to detection of two biomarkers, low density lipoprotein (LDL) and high density lipoprotein (HDL). They are the important biomarkers for diagnosing the cardiovascular diseases [16]. The immobilization of antibody onto the gold nanoparticle array was confirmed by observing the change of the LSPR peak. In addition, we showed the applicability of the gold nanoparticle array to the detection of biomarkers by measuring the change of the LSPR peak with the increasing concentration of the biomarkers.

\section{Materials and Methods}

2.1. Materials. LDL and antibody against LDL and HDL and antibody against HDL were purchased from Abcam plc company. 11-mercaptoundecanoic acid (11-MUA), 1-ethyl-3-(3dimethylaminopropyl)carbodiimide hydrochloride (EDAC), and human serum were purchased from Sigma-Aldrich company. The other chemicals used in this study were reagent grade and were obtained commercially.

2.2. Fabrication of Gold Nanoparticle Array. The gold nanoparticle array was fabricated by the method reported previously [15]. After a glass substrate (size: $24 \times 24 \mathrm{~mm}$ ) was cleaned with the piranha solution $(1: 4$ of hydrogen peroxide and sulfuric acid), $1 \mu \mathrm{m}$ thick aluminum film was deposited by electron beam evaporation (EI-5, ULVAC Co., USA). A two-step anodization process was executed to form the alumina mask on the substrate as described elsewhere $[17,18]$. In the first anodization process, after the substrate and the carbon electrode were placed in a beaker of $0.3 \mathrm{M}$ oxalic acid, $40 \mathrm{~V}$ was applied between the substrate and the carbon electrode to anodize the aluminum film for 13 minutes at $5^{\circ} \mathrm{C}$. The alumina film formed on the substrate was removed by immersing it in a solution of $6 \mathrm{wt} \% \mathrm{H}_{3} \mathrm{PO}_{4}$ and $1.5 \mathrm{wt} \%$ $\mathrm{CrO}_{3}$ at $60^{\circ} \mathrm{C}$. After the substrate was rinsed and dried, the second anodization process was executed with the aluminum

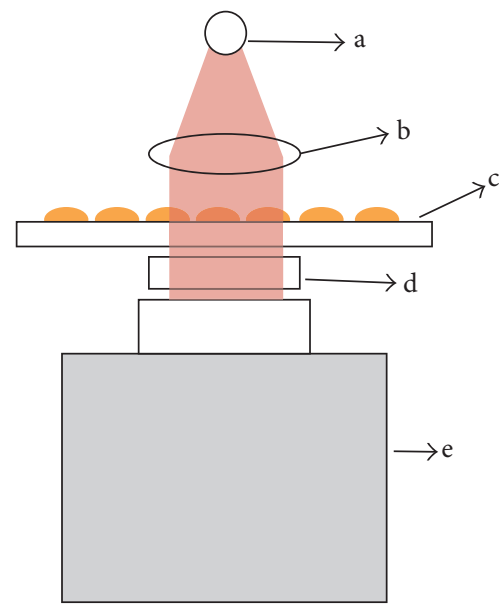

Figure 1: Hand-made equipment for measuring the optical transmission of substrate (a: halogen lamp, b: collimator, c: substrate with gold nanodot array, d: optical attenuator, and e: CCD-type spectrophotometer).

film remaining on the substrate under the same conditions as the first anodization. Finally, the substrate was immersed in a beaker of $5 \mathrm{wt} \% \mathrm{H}_{3} \mathrm{PO}_{4}$ to remove the barrier layer and widen the diameter of the pores formed in the alumina film [19].

The gold nanoparticle array was fabricated using the alumina mask lift-off process. To fabricate the gold nanoparticle array, a $2 \mathrm{~nm}$ thick layer of chromium and a gold layer were sequentially deposited via electron beam evaporation onto the substrate. After depositing the gold layer, the gold nanoparticle array on the substrate was completed by removing the alumina mask by immersing the substrate in $5 \mathrm{wt} \% \mathrm{H}_{3} \mathrm{PO}_{4}$ at $30^{\circ} \mathrm{C}$ for 60 minutes.

2.3. Immobilization of Antibody. The antibodies against biomarkers were covalently immobilized on the surface of each gold nanoparticle by the method reported elsewhere [20]. The glass substrate with the gold nanoparticle array was cleaned with ethanol, followed by the oxygen plasma treatment. The monolayer of 11-mercaptoundecanoic acid (11-MUA) was deposited on the gold nanoparticle array by submersing the substrate in the ethanol solution containing $150 \mathrm{mM}$ 11-MUA for at least 12 hours. To bind the antibody covalently, the carboxylic group of the monolayer was activated by submerging the substrate modified with 11-MUA into a solution of $10 \%$ EDAC in water/ethanol $(10 / 1 \mathrm{v} / \mathrm{v})$ for 2 hours at room temperature. The immobilization process of antibody was completed by applying the activated surface to $20 \mu \mathrm{g} / \mathrm{mL}$ antibody solution in the phosphate buffer saline (PBS) buffer for at least 2 hours, followed by cleaning the surface.

2.4. Experimental Setup. Absorption spectrums of LSPR substrate were acquired with the hand-made device equipped with the commercial components as shown in Figure 1. The device is composed of the CCD-array type spectrophotometer (BRC115A, B\&W Tek Inc.) and the $10 \mathrm{~W}$ tungsten lamp (Osram Co.) connected with the collimator and configured to 


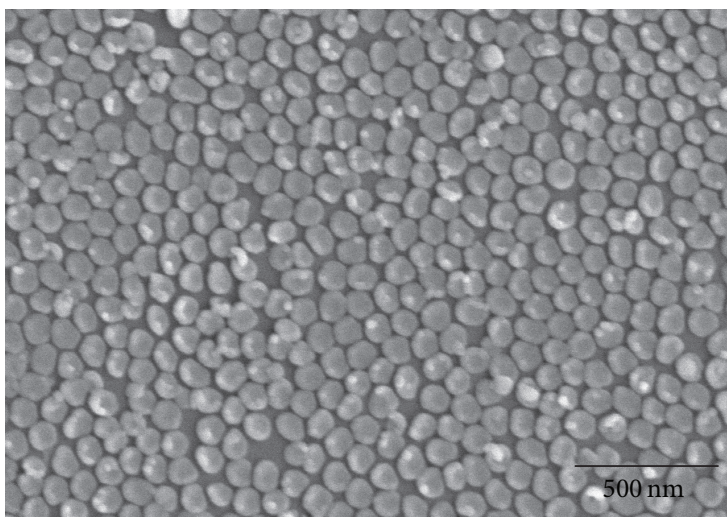

(a)

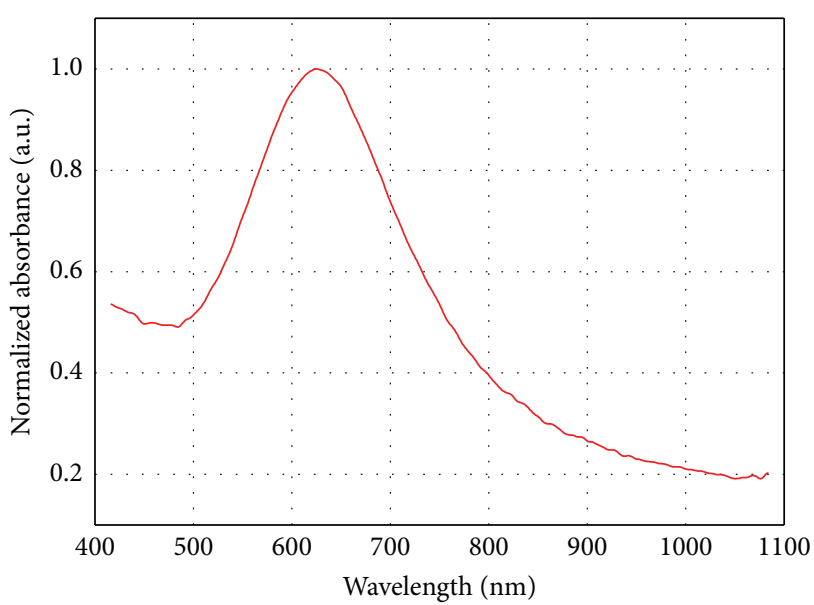

(b)

FIgURE 2: (a) Scanning electron micrograph of $10 \mathrm{~nm}$ thick gold nanodot array formed on glass substrate. (b) Typical absorption spectrum of the substrate with gold nanodot array.

measure the transmission of the substrate. The transmission value measured was converted into the absorbance value by Beer's law.

\section{Results and Discussion}

3.1. Gold Nanoparticle Array. As mentioned above, the gold nanoparticle array on the glass substrate was fabricated with the lift-off process of the alumina mask [15]. Previously, we reported that the sensitivity of LSPR substrate defined as the ratio of the shift of LSPR peak to the refractive index unit (RIU) of the medium surrounding the gold nanoparticle array was changed with the thickness of gold layer deposited, and the maximum sensitivity was at the deposition thickness of $10 \mathrm{~nm}$. Here, we used the gold nanoparticle array with the deposition thickness of $10 \mathrm{~nm}$ for the LSPR biosensing as shown in Figure 2(a). The diameter of each gold nanoparticle was $94.9 \pm 5.9 \mathrm{~nm}$, and the typical absorption spectrum of the substrate was shown in Figure 2(b), in which the position of LSPR peak of the gold nanoparticle array was $626.7 \pm 5.9 \mathrm{~nm}$, which is calculated with the centroid algorithm [21].

3.2. Immobilization of Antibody. Several techniques for binding antibody to the substrate have been reported, and are divided into two categories: the physical binding and the chemical binding. The chemical binding technique, in which antibody is covalently immobilized onto the substrate, has been found to show good reproducibility and coverage [22]. In this study, the chemical binding in which the monolayer of 11-MUA was used as the linker to bind antibody was used in order to immobilize the antibody on the gold nanoparticle array. Figure 3 shows the scheme of the immobilization of antibody used in this study. The monolayer of 11-MUA with the carboxylic acid as the terminal group was selfassembled on the surface of each gold nanoparticle. The substrate modified with the 11-MUA monolayer was treated with EDAC to allow the chemical binding between the lysine residues of antibody and the carboxylic group via the amide

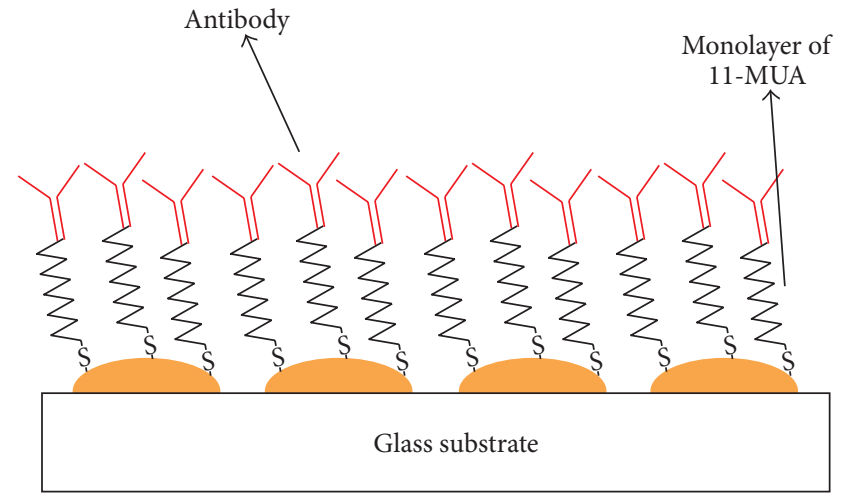

FIGURE 3: Scheme for immobilizing the antibody on the substrate.

bonding. Figures 4(a) and 4(b) show the change of absorption spectrums according to sequentially depositing the 11-MUA and antibody onto the substrate. Following the deposition of the monolayer of 11-MUA, the position of LSPR peak was shifted from $626.7 \pm 5.9 \mathrm{~nm}$ (the LSPR peak position of substrate without the monolayer) to $641.6 \pm 4.3 \mathrm{~nm}$. It is known that the closely packed 2-dimensional molecular layer of 11-MUA is formed on the substrate by the van der Waals attractive force among the long alkyl chains [23]. Therefore, the shift of peak position is attributed to the change of RIU near the gold nanoparticle array. After immobilizing the antibody against LDL onto the monolayer of 11-MUA, the LSPR peak was shifted to $676.8 \pm 4.2 \mathrm{~nm}$. Also, in case of immobilization of antibody against HDL, the LSPR peak of substrate was shifted to $671.1 \pm 5.6 \mathrm{~nm}$.

3.3. Detection of Biomarkers. LDL is the major carrier of cholesterol in the blood, and the accumulation of it on the vessel wall constitutes one of the initial steps in arteriosclerosis [24]. So, the increased level of LDL in blood is associated with atherosclerosis. On the other hand, HDL is 


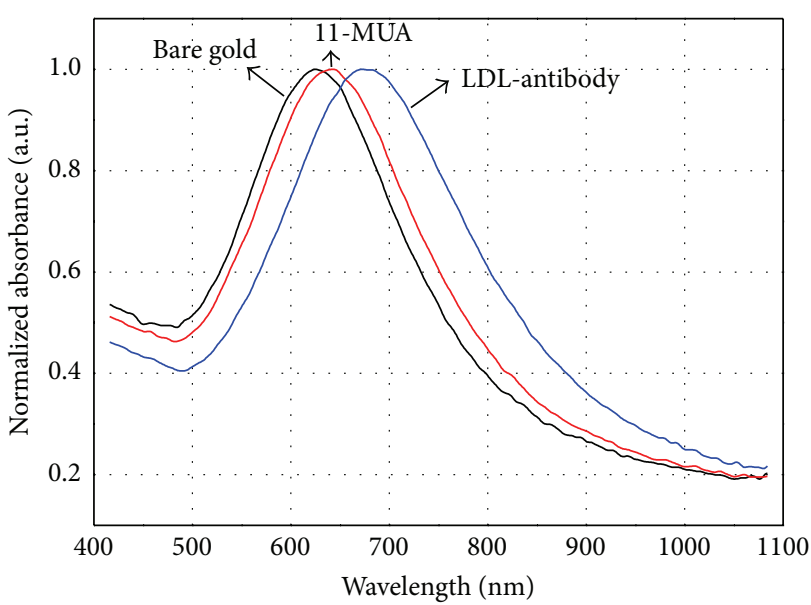

(a)

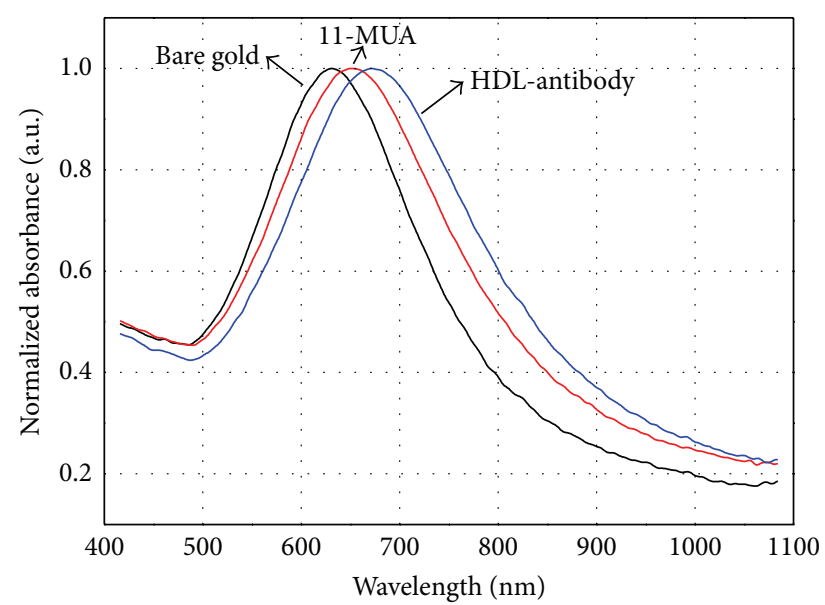

(b)

FIGURE 4: Absorption spectrums of substrate with depositing the 11-MUA monolayer and immobilizing the antibodies on the monolayer. (a) Immobilization of the antibody against LDL. (b) Immobilization of the antibody against HDL.

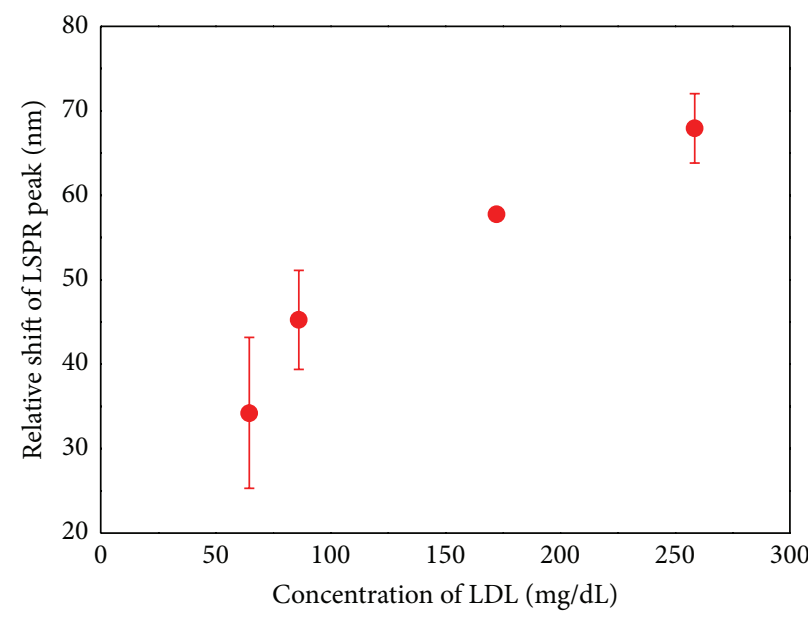

(a)

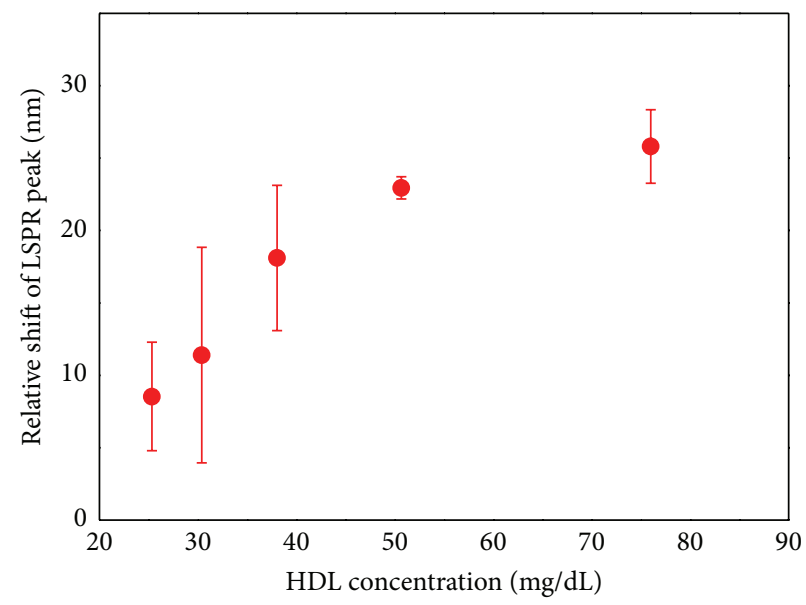

(b)

FIGURE 5: Relative shifts of LSPR peak corresponding with reacting with the varying concentration of biomarkers: LDL (a) and HDL (b).

the lipoprotein which has been shown to be able to prevent and reverse arteriosclerosis [16]. So, the level of HDL in blood should be higher than the normal concentration level. Therefore, as LDL and HDL are the import biomarkers to diagnose or monitor the cardiovascular diseases, the detections of them are routinely executed in the laboratory medicine. Here, the detection of LDL and HDL with the LSPR of the gold nanoparticle array substrate was demonstrated.

In the experiment, the LDL and HDL were diluted into the varying concentrations in the human serum purchased from Sigma-Aldrich Co. Then, the substrate with the antibody immobilized was incubated in the biomarker solutions with the varying concentration for 1 hour with gently stirring. After being incubated, the substrate was cleaned with the water, followed by drying under the nitrogen gas. The absorption spectrum of substrate was acquired with the equipment described above. Figure 5(a) shows the relative shift of LSPR peak as a function of the concentration of LDL. The relative shift of LSPR peak in the vertical axis of the graphs meant the amount of the shift of LSPR peak from the LSPR peak of substrate with the antibody immobilized. The concentrations of LDL prepared in the experiment were $64.6,86.2,172.3$, and $258.5 \mathrm{mg} / \mathrm{dL}$, respectively. In the range, the relative shift of LSPR peak increased with the increasing concentration of LDL. According to the American Heart Association, the optimal level of LDL in blood is lower than $100 \mathrm{mg} / \mathrm{dL}$, and the value higher than $200 \mathrm{mg} / \mathrm{dL}$ is very high LDL level, corresponding to highest increased rates of cardiovascular disease event [25]. The experimental result shows that our LSPR substrate can be used to detect the LDL in the clinical available range of concentration. Figure 5(b) shows the relative shift of LSPR peak as a function of the concentration of HDL. Similar to the experiment of LDL detection, the LSPR peak was positively shifted with the 
increasing concentration of HDL. In particular, the relative shift of LSPR is linearly proportional to the concentration in the low concentration range. Contrary to the LDL level, it is known that the optimal level of HDL in blood is higher than $60 \mathrm{mg} / \mathrm{dL}$, and the values under $40 \mathrm{mg} / \mathrm{dL}$ for men and under $50 \mathrm{mg} / \mathrm{dL}$ for women are risky for heart diseases [25]. The experimental result shows the LSPR substrate with the gold nanoparticle array can be applied to detect the HDL.

In this study, we showed the applicability of the substrate with the gold nanoparticle array to detect the LDL and HDL. The LSPR substrate for the development of biosensor provides two advantages over the conventional analysis [26]. At first, the detection time is short because the step for labeling fluorophores or ELISA is omitted. Moreover, as the probe antibody used for labeling is not needed, it is costeffective. Second, the equipment for readout is simple. As described above, the antigen-antibody binding can be simply detected by measuring the optical transmission of substrate. In reality, we constructed the equipment for measuring the transmission with the engineering technique not complicated. Our gold nanoparticle array and its fabrication method provide the simple and powerful solution to develop the LSPR-based biosensor. However, there are several hurdles to overcome to apply the LSPR substrate in the clinical area. The sample for analyzing in the clinical diagnostics is the blood, which is the complex of white and red blood cells, and the serum with several proteins. In particular, as the high concentration of red blood cells in blood affects the measurement of transmission, only the plasma should be extracted from blood before analyzing the biomarker. Recently, the microfluidics technology for extracting the plasma from the blood has been reported [27, 28]. In our opinion, the point-of-care device for biosensing LDL and HDL can be developed with the strategy of allocating the LSPR substrate in the downstream of plasma in the microfluidic device.

\section{Conclusions}

In this study, we showed the feasibility of the LSPR substrate with gold nanoparticle array to detect LDL and HDL, which are the biomarkers for diagnosing and monitoring the cardiovascular diseases. The LSPR substrate was fabricated through the lift-off process with the anodized alumina mask, and its LSPR phenomenon was observed by measuring the optical transmission of substrate. The antibodies were immobilized on the gold nanoparticle array via the chemical binding, in which the 11-MUA was used as the linker to bind the antibodies. The binding of antibodies was confirmed by observing the shift of LSPR peak of the substrate. Finally, with the LSPR substrates with the antibodies immobilized, the detection of LDL and HDL was investigated. As a result, LDL and HDL could be detected in the clinically available concentration range, respectively.

When the LSPR substrate is used for the development of biosensor, it provides the advantages such as the fast detection protocol and the cost-effectiveness. In particular, by fusing the LSPR substrate and the microfluidic device, the development of point-of-care diagnostic device based on the immunoassay can be implemented in the view of practice.

\section{Conflict of Interests}

The authors declare that there is no conflict of interests regarding the publication of this paper.

\section{Acknowledgment}

This research was supported by Korea Electrotechnology Research Institute (KERI) Primary Research Program through the Korea Research Council for Industrial Science \& Technology (ISTK) funded by the Ministry of Science, ICT and Future Planning (MSIP) (no. 15-12-N0101-29).

\section{References}

[1] L. Gervais and E. Delamarche, "Toward one-step point-of-care immunodiagnostics using capillary-driven microfluidics and PDMS substrates," Lab on a Chip, vol. 9, no. 23, pp. 3330-3337, 2009.

[2] H. Xie, J. Mire, Y. Kong et al., "Rapid point-of-care detection of the tuberculosis pathogen using a BlaC-specific fluorogenic probe," Nature Chemistry, vol. 4, no. 10, pp. 802-809, 2012.

[3] E. Stern, A. Vacic, N. K. Rajan et al., "Label-free biomarker detection from whole blood," Nature Nanotechnology, vol. 5, no. 2, pp. 138-142, 2010.

[4] Y. M. Bae, S. Y. Son, K.-H. Lee, K. Kim, S. K. Seol, and D. Kim, "Enhancement of the sensitivity of surface plasmon resonance biosensor by modifying the metal surface," Journal of Biomedical Nanotechnology, vol. 9, no. 6, pp. 1060-1064, 2013.

[5] G. Wu, R. H. Datar, K. M. Hansen, T. Thundat, R. J. Cote, and A. Majumdar, "Bioassay of prostate-specific antigen (PSA) using microcantilevers," Nature Biotechnology, vol. 19, no. 9, pp. 856860, 2001.

[6] Y. Tang, R. Memaugh, and X. Zeng, "Nonregeneration protocol for surface plasmon resonance: study of high-affinity interaction with high-density biosensors," Analytical Chemistry, vol. 78, no. 6, pp. 1841-1848, 2006.

[7] Y.-B. Shin, H. M. Kim, Y. Jung, and B. H. Chung, "A new palm-sized surface plasmon resonance (SPR) biosensor based on modulation of a light source by a rotating mirror," Sensors and Actuators, B: Chemical, vol. 150, no. 1, pp. 1-6, 2010.

[8] K. A. Willets and R. P. Van Duyne, "Localized surface plasmon resonance spectroscopy and sensing," Annual Review of Physical Chemistry, vol. 58, pp. 267-297, 2007.

[9] K. L. Kelly, E. Coronado, L. L. Zhao, and G. C. Schatz, "The optical properties of metal nanoparticles: the influence of size, shape, and dielectric environment," Journal of Physical Chemistry B, vol. 107, no. 3, pp. 668-677, 2003.

[10] D. A. Gish, F. Nsiah, M. T. McDermott, and M. J. Brett, "Localized surface plasmon resonance biosensor using silver nanostructures fabricated by glancing angle deposition," Analytical Chemistry, vol. 79, no. 11, pp. 4228-4232, 2007.

[11] J. N. Anker, W. P. Hall, O. Lyandres, N. C. Shah, J. Zhao, and R. P. Van Duyne, "Biosensing with plasmonic nanosensors," Nature Materials, vol. 7, no. 6, pp. 442-453, 2008.

[12] C. L. Haynes and R. P. Van Duyne, "Nanosphere lithography: a versatile nanofabrication tool for studies of size-dependent nanoparticle optics," Journal of Physical Chemistry B, vol. 105, no. 24, pp. 5599-5611, 2001. 
[13] S.-W. Lee, K.-S. Lee, J. Ahn, J.-J. Lee, M.-G. Kim, and Y.-B. Shin, "Highly sensitive biosensing using arrays of plasmonic Au nanodisks realized by nanoimprint lithography," ACS Nano, vol. 5, no. 2, pp. 897-904, 2011

[14] M. E. Stewart, C. R. Anderton, L. B. Thompson et al., "Nanostructured plasmonic sensors," Chemical Reviews, vol. 108, no. 2, pp. 494-521, 2008.

[15] Y. M. Bae, K.-H. Lee, J. Yang, and D. Heo, "Fabrication of gold nanodot array for the localized surface plasmon resonance," Journal of Nanomaterials, vol. 2014, Article ID 175670, 7 pages, 2014.

[16] G. Siegel, M. Malmsten, D. Klüßendorf, and F. Michel, "A receptor-based biosensor for lipoprotein docking at the endothelial surface and vascular matrix," Biosensors and Bioelectronics, vol. 16, no. 9-12, pp. 895-904, 2001.

[17] H. Masuda and K. Fukuda, "Ordered metal nanohole arrays made by a two-step replication of honeycomb structures of anodic alumina," Science, vol. 268, no. 5216, pp. 1466-1468, 1995.

[18] K. Nielsch, J. Choi, K. Schwirn, R. B. Wehrspohn, and U. Gösele, "Self-ordering regimes of porous alumina: the $10 \%$ porosity rule," Nano Letters, vol. 2, pp. 677-680, 2002.

[19] B. Yan, H. T. M. Pham, Y. Ma, Y. Zhuang, and P. M. Sarro, "Fabrication of in situ ultrathin anodic aluminum oxide layers for nanostructuring on silicon substrate," Applied Physics Letters, vol. 91, no. 5, Article ID 053117, 2007.

[20] Y. M. Bae, B.-K. Oh, W. Lee, W. H. Lee, and J.-W. Choi, "Immunosensor for detection of Yersinia enterocolitica based on imaging ellipsometry," Analytical Chemistry, vol. 76, no. 6, pp. 1799-1803, 2004.

[21] S. Zhan, X. Wang, and Y. Liu, "Fast centroid algorithm for determining the surface plasmon resonance angle using the fixedboundary method," Measurement Science and Technology, vol. 22, no. 2, Article ID 025201, 2011.

[22] Y. M. Bae, B.-K. Oh, W. Lee, W. H. Lee, and J.-W. Choi, "Study on orientation of immunoglobulin G on protein G layer," Biosensors and Bioelectronics, vol. 21, no. 1, pp. 103-110, 2005.

[23] A. Ulman, "Formation and structure of self-assembled monolayers," Chemical Reviews, vol. 96, no. 4, pp. 1533-1554, 1996.

[24] P. A. Serra, Biosensors for Detection of Low-Density Lipoprotein and Its Modified Forms in Biosensors for Health, Environment and Biosecurity, chapter 9, Intech, Rijeka, Croatia, 2011.

[25] S. M. Grundy, G. J. Balady, M. H. Criqui et al., "When to start cholesterol-lowering therapy in patients with coronary heart disease," Circulation, vol. 95, no. 6, pp. 1683-1685, 1997.

[26] Y. Hong, Y.-M. Huh, D. S. Yoon, and J. Yang, "Nanobiosensors based on localized surface plasmon resonance for biomarker detection," Journal of Nanomaterials, vol. 2012, Article ID 759830, 13 pages, 2012.

[27] V. VanDelinder and A. Groisman, "Separation of plasma from whole human blood in a continuous cross-flow in a molded microfluidic device," Analytical Chemistry, vol. 78, no. 11, pp. 3765-3771, 2006.

[28] K. K. Lee and C. H. Ahn, "A new on-chip whole blood/plasma separator driven by asymmetric capillary forces," Lab on a Chip, vol. 13, no. 16, pp. 3261-3267, 2013. 

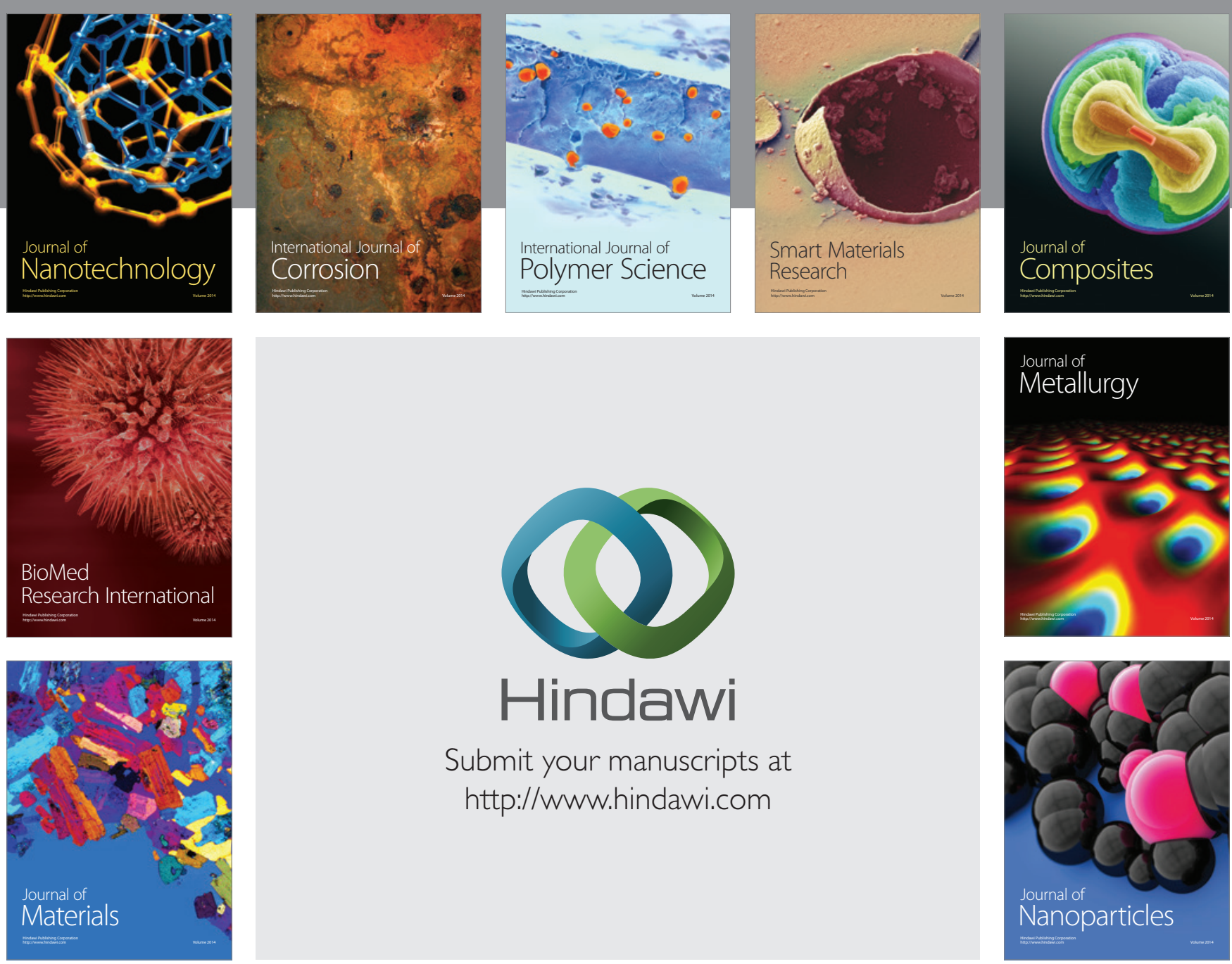

Submit your manuscripts at http://www.hindawi.com
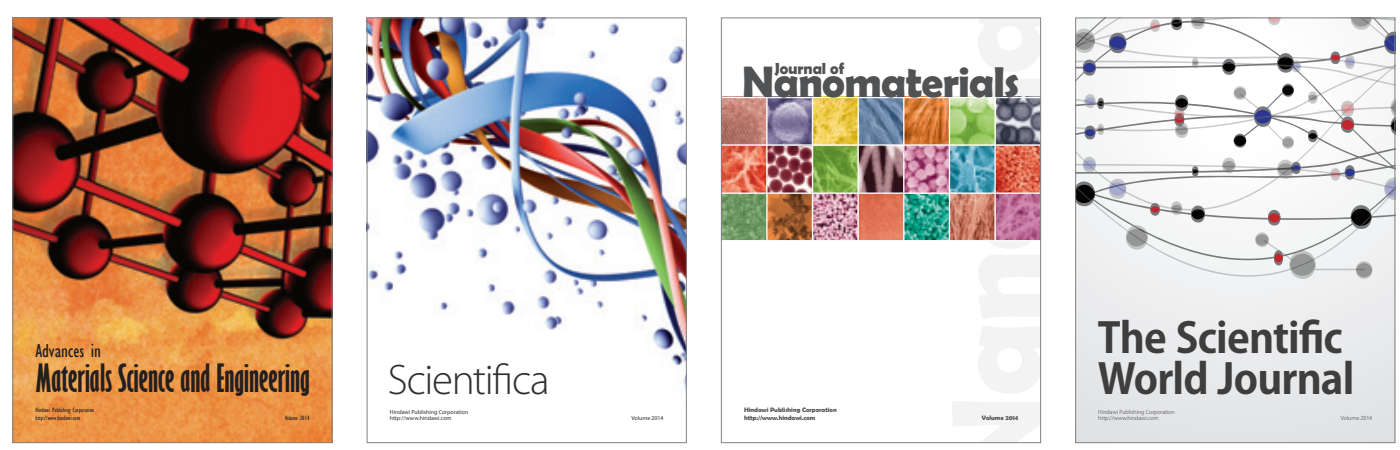

\section{The Scientific World Journal}
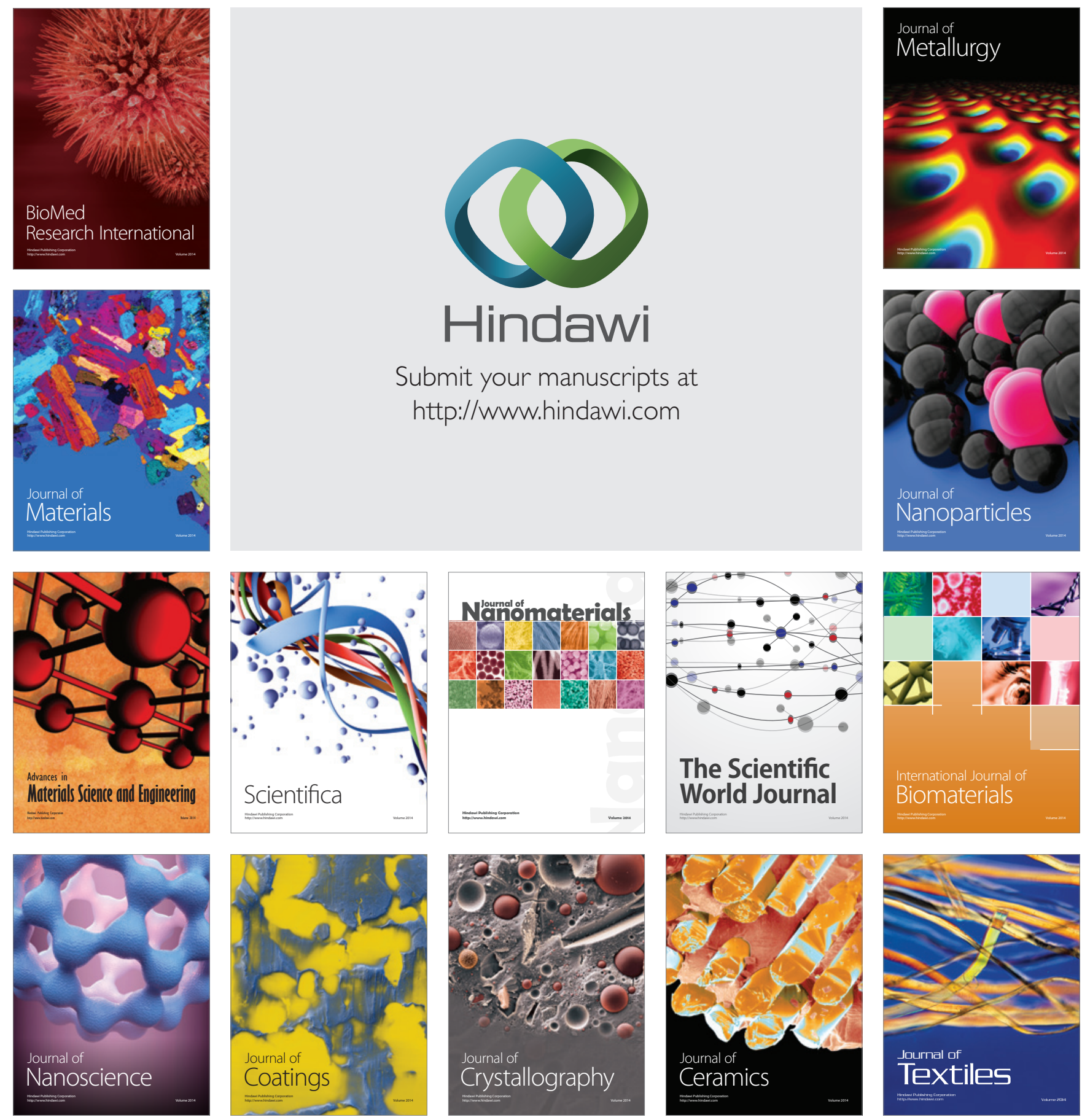